\title{
Toracoscopia nas hérnias diafragmáticas: estudo experimental em cães
}

\author{
Thoracoscopic techniques in diaphragmatic hernias: experimental study in dogs
}

\author{
Carlos Afonso de Castro Beck ${ }^{1}$ Ney Luis Pippi ${ }^{2}$ Maurício Veloso Brun ${ }^{3}$ \\ Emerson Antônio Contesini ${ }^{1}$ Anderson Favaro da Cunha ${ }^{5}$ Rafael Stedile $^{5}$ \\ Adamas Tassinari Bonfada ${ }^{5}$ Antonio de Pádua Ferreira da Silva Filho ${ }^{6}$ \\ Kleber Gomes ${ }^{7}$ Lucas Marques Colomé $^{7}$
}

RESUMO

Na classificação das hérnias diafragmáticas, a traumática é a forma mais prevalente em cães e gatos. A alteração, que se caracteriza por uma alteração anatômica, tem na correção cirúrgica, o tratamento recomendado. $O$ presente estudo se propôs a avaliar a cirurgia videotoracoscópica no diagnóstico e tratamento de hérnias diafragmáticas em cães. Na primeira etapa do experimento, oito animais foram submetidos à produção de um modelo de hérnia diafragmática por meio do acesso laparoscópico. Após o período de uma semana, os cães foram submetidos à segunda etapa experimental através do acesso toracoscópico, tendo como finalidade o diagnóstico e a terapêutica cirúrgica. $O$ acesso toracoscópico mostrou-se eficaz tanto no caráter diagnóstico das rupturas e dos deslocamentos, como na correção cirúrgica de reposição visceral e rafia diafragmática.

Palavras-chave: videotoracoscopia, hérnia diafragmática, cães.

\section{ABSTRACT}

The most common type of diaphragmatic hernia in dogs and cats is the traumatic one.As an anatomic alteration surgery is the elected treatment. The purpose of this study was to evaluate videothoracoscopic surgery in the diagnostic and treatment of diaphragmatic hernia in eight dogs. In the first phase of the study diaphragmatic hernias were performed in the dogs through laparoscopic access. One week later the dogs were summited to a thoracoscopic surgery to make diagnostic and surgical correction. Thoracoscopic access showed to be efficient for identification of the defects and visceral displacements and for the surgical treatment.

Key words: videothoracoscopic, diaphragmatic hernia, dog.

\section{INTRODUÇÃO}

A hérnia diafragmática decorrente de trauma é a forma mais prevalente entre as hérnias diafragmáticas em cães e gatos (LEVINE, 1987; JOHNSON, 1993; RAISER, 1993), sendo as decorrentes de acidente automobilístico as mais freqüente (WILSON et al., 1971; BOUDRIEAU \& MUIR, 1987; LEVINE, 1987; RAISER, 1994), seguidas de quedas, chutes e brigas (BOUDRIEAU \& MUIR, 1987; LEVINE, 1987; HAGE \& IWASAKI, 2001).

O conteúdo herniário pode variar conforme a extensão da ruptura e a atividade do animal (KIRK \& BISTNER, 1987). Estudos de achados cirúrgicos relacionaram pela ordem o fígado, o intestino delgado, o estômago, o baço e o omento, como as vísceras que se deslocam com maior freqüência para o tórax (BOUDRIEAU \& MUIR, 1987; RAISER, 1993).

Em decorrência da alteração presente nas rupturas diafragmáticas ser de natureza anatômica, o tratamento cirúrgico é o único indicado (RAISER, 1993;

\footnotetext{
${ }^{1}$ Médico Veterinário. Doutor. Professor Assistente do Departamento de Medicina Animal, Faculdade de Veteinária, Universidade Federal do Rio Grande do Sul (UFRGS). Av. Bento Gonçalves, 9090, 91540-000, Porto Alegre, RS, Brasil. E-mail: afonso@orion.ufrgs.br.

${ }^{2}$ Médico Veterinário, PhD, Professor Adjunto, Departamento de Clínica de Pequenos Animais, Universidade Federal de Santa Maria (UFSM).

${ }^{3}$ Médico Veterinário, Doutor, Professor do Curso de Medicina Veterinária da Universidade de Passo Fundo (UPF).

${ }^{4}$ Médico Veterinário, Mestre.

${ }^{5}$ Aluno do Curso de Mestrado em Medicina Veterinária na área de Cirurgia Experimental, UFSM.

${ }^{6}$ Médico Veterinário, Doutor, Professor Adjunto, Departamento de Medicina Animal, Faculdade de Veterinária, UFRGS

${ }^{7}$ Aluno do Curso de Medicina Veterinária da UFSM, Bolsista de Iniciação Científica.
} 
HAGE \& IWASAKI, 2001). Entretanto, é fundamental que o paciente esteja estável antes de ser submetido à intervenção cirúrgica. A cirurgia apenas será realizada em caráter emergencial, quando existir sério comprometimento cardiorespiratório, representando risco de vida (JOHNSON, 1993; RAISER, 1993). As abordagens cirúrgicas mais utilizadas são a laparotomia pela linha média e a toracotomia intercostal. A escolha será determinada pela capacidade de localizar o lado da ruptura e a sua cronicidade, com relação às aderências torácicas (JOHNSON, 1993).

A cirurgia endoscópica é caracterizada como um acesso cirúrgico minimamente invasivo, no qual a visibilização é realizada através de um endoscópio. É também denominada de cirurgia laparoscópica, celioscópica, ou pelvioscópica, quando o procedimento envolve o acesso abdominal, e toracoscópica, pleuroscópica, ou cirurgia toracoscópica vídeo-assistida (CTVA), quando o procedimento envolve o acesso torácico (FREEMAN, 1998).

A toracoscopia foi originalmente introduzida por Jacobeus, em 1910, quando o pesquisador usou um cistoscópio modificado, sem magnificação de imagens, para realizar diagnósticos de tuberculose. Na última década, o acesso toracoscópico tem registrado uma expansão importante, quer nas possibilidades diagnósticas, quer nas terapêuticas (JAKLITSCH et al., 1996). A partir do início da década de 90, a técnica foi utilizada em maior escala, sendo empregada com êxito na identificação de efusão pleural, de fístulas bronco-pleurais, de lesões esofágicas, de anormalidades pericárdicas, de doenças pulmonares intersticiais difusas e de nódulos pulmonares (CARRILO et al., 1997). Da mesma maneira, o acesso passou a ser utilizado com objetivos terapêuticos, como na ressecção de nódulos pulmonares (NAKAJIMA et al., 2000), excisão e drenagem pericárdica, pleurodesis e biópsias pulmonares (MACK et al. 1992) e drenagem de abscesso mediastinal (CHUNG \& RITCHIE, 2000).

Em trabalho de revisão, REMEDIOS \& FERGUSON (1996) consideraram ainda limitado o acesso toracoscópico na medicina veterinária. Os estudos iniciais restringiram-se à exploração diagnóstica da cavidade (McCARTHY \& McDERMID, 1990). Porém, ultimamente a toracoscopia vem ganhando terreno também na Medicina Veterinária, sendo utilizada experimentalmente nas biópsias pulmonares, lobectomias (GARCÍA et al., 1998) e pericardiectomias (WALSH et al., 1999) em cães. WALTON (2001) acrescentou que a toracoscopia é instrumento importante para determinação de estágios tumorais, visualização de biópsias, avaliação e tratamento de pneumotórax persistente, tratamento de efusão pericárdica e pleural e na avaliação e reparo do trauma intratorácico em pequenos animais.

SMITH et al. (1993) realizaram cirurgias toracoscópicas em 24 pacientes humanos com trauma torácico e destacaram que a técnica mostrou-se segura, acurada e com mínimo trauma operatório. De acordo com o estudo, a CTVA evitou a celiotomia em 15 dos 24 pacientes $(63 \%)$. Os autores consideraram a hemorragia contínua, a remoção de coágulos torácicos e as lacerações diafragmáticas como as principais indicações da técnica.

A principal contra-indicação da CTVA no trauma diz respeito à instabilidade hemodinâmica do paciente, sendo relacionadas, ainda, a suspeita de lesões críticas ao coração e aos grandes vasos, a obliteração do espaço pleural, a toracotomia anterior, as indicações para toracotomia de emergência e a inabilidade para tolerar a ventilação seletiva (LOWDERMILK \& NAUNHEIM, 2000).

Em estudo experimental, BRUN et al. (1998) realizaram o reparo de lacerações diafragmáticas através do acesso toracoscópico em cinco cães e concluíram que o mesmo permitiu a terapêutica cirúrgica no modelo animal desenvolvido.

O presente estudo objetivou avaliar o acesso toracoscópico no diagnóstico e na terapêutica cirúrgica de hérnias diafragmáticas produzidas experimentalmente em cães.

\section{MATERIAL E MÉTODOS}

No presente estudo, foram utilizados oito cães adultos hígidos, machos, sem raça definida, oriundos do Biotério Central da UFSM, com pesos que variaram entre 11 e $33 \mathrm{~kg}$. Previamente à inclusão no experimento os animais foram avaliados clinicamente, everminados e alojados em canis coletivos onde receberam, ração comercial e água $a d$ libitum.

O experimento foi dividido em duas etapas. Na primeira etapa, os animais foram submetidos a um procedimento cirúrgico com o objetivo de induzir um modelo de hérnia diafragmática através do acesso laparoscópico. Em quatro cães, a indução da ruptura foi do lado direito e nos restantes do lado esquerdo. $\mathrm{O}$ protocolo anestésico constou de medicação préanestésica (MPA) com associação de cetamina (6,0mg $\left.\mathrm{kg}^{-1}\right)$ e midazolam $\left(0,3 \mathrm{mg} \mathrm{kg}^{-1}\right)$, via IM, na mesma seringa. Na indução anestésica, foi utilizada cetamina $\left(5,0 \mathrm{mg} \mathrm{kg}^{-1}\right)$, via IV, e, na manutenção, isofluorano vaporizado em oxigênio a $100 \%$ em circuito 
semifechado com respiração mecanicamente controlada.

O procedimento teve início através de punção com agulha de Veress, próximo à cicatriz umbilical, e estabelecimento do pneumoperitônio utilizando uma pressão de $11 \mathrm{mmHg}$. Na seqüência foram introduzidos os três trocartes, respectivamente para a inserção do endoscópio e para os instrumentos de trabalho. Através do segundo trocarte, foi inserido um segmento de equipo com $6 \mathrm{~cm}$ de extensão, sendo este adaptado entre a porção esternal e costal do diafragma, no sentido circunferencial, sendo na seqüência realizada a demarcação do local da ruptura diafragmática. Imediatamente antes de promover a ruptura do diafragma, o pneumoperitônio foi reduzido de 11 para $5 \mathrm{mmHg}$, com o objetivo de diminuir os riscos de um pneumotórax compressivo. O defeito diafragmático, previamente delimitado em $6 \mathrm{~cm}$, foi estabelecido com a utilização de uma tesoura de Metzenbaum. Ao final do procedimento, o instrumental cirúrgico e os trocartes foram removidos, os locais de punção foram obliterados e a pressão torácica negativa foi restabelecida através de punção torácica com cateter venoso adaptado a uma válvula de três vias e seringa de $100 \mathrm{ml}$.

A segunda etapa foi realizada uma semana após a primeira, tendo como objetivo inicial identificar a ruptura diafragmática e/ou a presença de vísceras abdominais no tórax e, em seguida, promover a terapêutica cirúrgica de redução do conteúdo herniário e a síntese do diafragma. Nesta segunda etapa, o acesso cirúrgico utilizado foi o toracoscópico.

Após a preparação pré-operatória e indução anestésica, os cães foram posicionados em decúbito lateral com o lado da ruptura diafragmática voltado para cima. O posicionamento da equipe cirúrgica, do animal, dos equipamentos e instrumental cirúrgico, encontram-se ilustrados na figura $1 \mathrm{~A}$. O tórax sofreu acesso pelo método aberto, com o intuito de reduzir o risco de lesões iatrogênicas às estruturas torácicas. Uma pequena incisão de aproximadamente $2 \mathrm{~cm}$ de extensão foi realizada no terço dorsal do $6^{\circ}$ ou $7^{\circ}$ espaço intercostal, alcançando a pele, tecido subcutâneo e muscular, além da pleura parietal. Pela abertura, foi introduzida a cânula do primeiro trocarte de $11 \mathrm{~mm} \varnothing$, adaptando-se o manguito do insuflador automático à válvula da cânula. A pressão utilizada na insuflação do tórax foi de $5 \mathrm{~mm} \mathrm{Hg}$ de $\mathrm{CO}_{2}$. Pelo interior desta cânula, foi introduzido o endoscópio rígido de $0^{\circ}$ e 10 $\mathrm{mm} \emptyset$.

A partir deste momento, teve início a exploração torácica, denominada como procedimento diagnóstico. A localização da ruptura diafragmática e/ ou identificação de vísceras abdominais, presentes no tórax, caracterizaram o procedimento como positivo. Além destes achados, foram registradas quaisquer alterações observadas no interior do tórax, como aspecto das vísceras abdominais deslocadas, colabamento pulmonar, presença de aderências, derrame pleural, entre outros. Na seqüência, teve início a terapêutica cirúrgica. O primeiro passo constou da escolha dos outros dois locais de punção. O segundo trocarte foi introduzido no $5^{\circ}$ ou $6^{\circ}$ espaço intercostal, próximo à junção costo-condral e o terceiro trocarte no 7o ou 8e espaço intercostal, no seu terço médio. As variações de localização dos trocartes obedeceram às variações de conformação anatômica dos cães. A disposição dos trocartes está representada na Figura $1 \mathrm{~B}$.

A toracoscopia teve seguimento com o início das manobras de reposicionamento das vísceras abdominais. Nestas manobras, foram utilizadas pinça atraumática Babcock, pinça de Maryland, pinça de Kelly e afastador de vísceras articulado, através dos dois trocartes de trabalho. Após a redução visceral, iniciou-se a síntese da ruptura diafragmática. Um segmento de fio poliglactina 910 agulhado (no3-0), previamente medido em $20 \mathrm{~cm}$, foi introduzido no tórax por meio de um redutor de diâmetro, adaptado à cânula do $2^{\mathrm{o}}$ trocarte. Com o uso de um porta-agulhas endoscópico e pinça de Kelly, foi realizada a síntese do diafragma, utilizando-se sutura contínua simples. Ao final foi realizada a inspeção da cavidade, sendo promovida a drenagem da efusão pleural, quando presente no tórax. As cânulas do $2^{\circ}$ e $3 \stackrel{0}{\circ}$ trocartes foram removidas. Os locais das duas punções foram obliterados por meio de sutura de Wolff com fio poliglactina-910 nำ3-0, para a musculatura intercostal e mononailon 3-0 em padrão isolado simples para a pele. A entrada de $\mathrm{CO}_{2}$ foi suspensa, a cânula do $1^{\mathrm{o}}$ trocarte removida e, após expansão prolongada e completa dos pulmões, o local da primeira punção foi suturado de maneira semelhante aos demais. O pneumotórax residual foi drenado com o auxílio de uma seringa de $100 \mathrm{ml}$ adaptada à válvula de três vias e cateter venoso.

\section{RESULTADOS}

O acesso laparoscópico foi efetivo, permitindo a indução da ruptura diafragmática, esquerda ou direita, nos oito cães. O tempo cirúrgico total desta etapa variou de 21 a 44 minutos, com tempo médio de 28,37 minutos. A abordagem possibilitou a visualização do diafragma, das estruturas e das vísceras abdominais. Não houve diferença entre os 


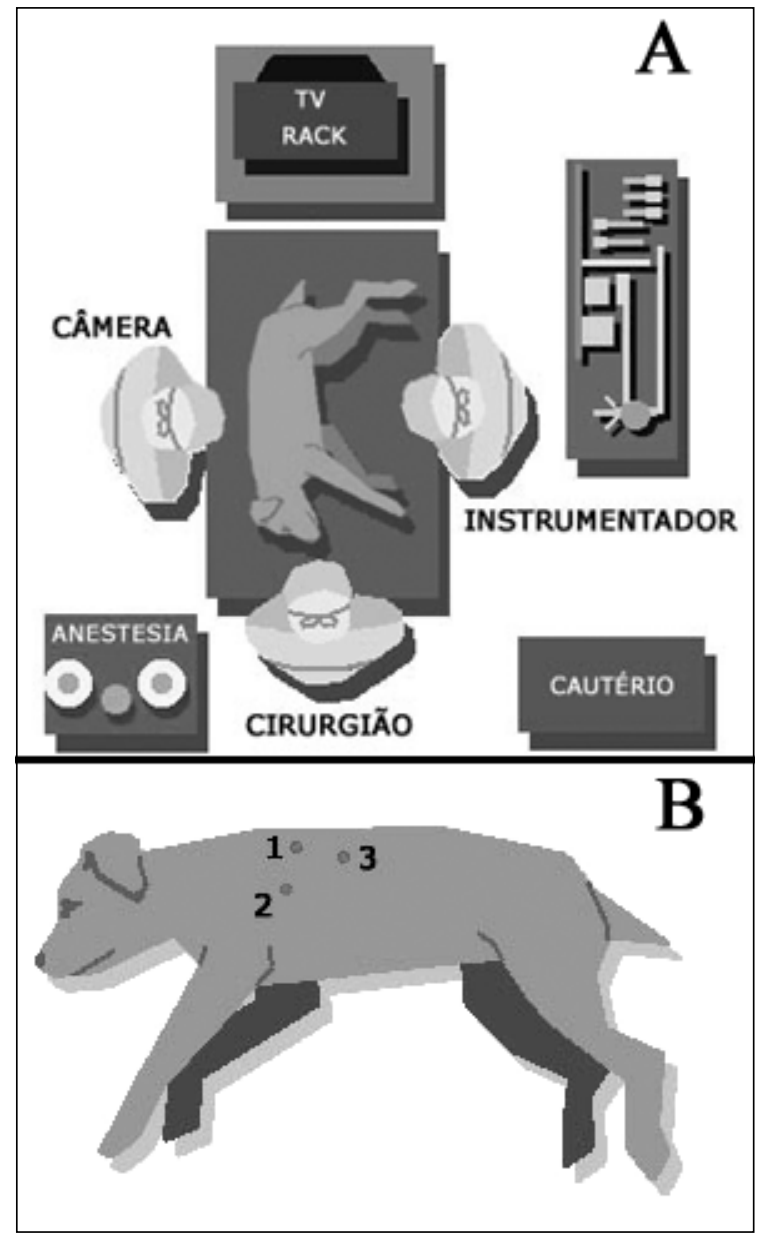

Figura 1 - Disposição da equipe cirúrgica e equipamentos durante os procedimentos toracoscópicos (A). Localização dos 3 trocartes em distribuição triangular durante as toracoscopias (B).

lados direito e esquerdo quanto à identificação do diafragma e à promoção de sua ruptura.

O segmento de equipo de $6 \mathrm{~cm}$ de extensão foi facilmente introduzido no interior da cavidade abdominal através de um redutor de diâmetro utilizado no interior da cânula do segundo trocarte, sendo na seqüência adaptado à superfície côncava da face abdominal do diafragma. A redução da pressão de $\mathrm{CO}$ do pneumoperitônio, de $11 \mathrm{~mm}$ para $5 \mathrm{mmHg}$, antes dâ abertura diafragmática, evitou a ocorrência de pneumotórax compressivo durante as manobras cirúrgicas da $1^{\underline{a}}$ etapa. Durante o intervalo de uma semana entre a $1^{\mathfrak{a}}$ e a $2^{\underline{a}}$ etapa, dois cães manifestaram sinais leves de dispnéia, acrescidos de prostração e de inapetência.

As manobras relacionadas à $2^{\underline{a}}$ etapa do experimento resultaram em um tempo cirúrgico total médio de $94,14 \pm 38,27$. O acesso toracoscópico mostrou-se efetivo, permitindo a identificação da ruptura diafragmática, esquerda ou direita, nos oito cães. O posicionamento da equipe cirúrgica, bem como o da torre com os equipamentos vídeo-endoscópicos, o da mesa cirúrgica e o dos animais mostrou-se adequado para a realização dos procedimentos cirúrgicos. A utilização do método aberto para introdução do primeiro trocarte foi satisfatória, sem a ocorrência de qualquer lesão iatrogênica durante a manobra. O pneumotórax, utilizando $5 \mathrm{~mm}$ de $\mathrm{Hg}$ de $\mathrm{CO}$, foi adequado, embora no caso de três cães tenha sidó necessário, num ou noutro momento cirúrgico, suspender a insuflação da cavidade para que alterações cardiorrespiratórias fossem corrigidas.

A abordagem toracoscópica lateral possibilitou uma adequada visualização do hemidiafragma correspondente, das estruturas e dos órgãos torácicos, além das vísceras abdominais, sem diferença no que se refere aos lados direito e esquerdo. $\mathrm{O}$ procedimento diagnóstico, realizado a partir da introdução do endoscópio, através da cânula do primeiro trocarte, foi considerado positivo para todos os cães. Entre as vísceras abdominais presentes no interior do tórax e de acordo com o número de animais, observou-se: o fígado (7), o omento (5), o intestino delgado (2) e o estômago (2) (Figura 2). Além do deslocamento das vísceras abdominais, foi possível identificar aderência da pleura a lobos hepáticos (2), aderência da pleura às bordas da ruptura do diafragma (2), aderência de lobos hepáticos às bordas da ferida diafragmática (5), presença de efusão pleural (4) e colabamento parcial de lobo pulmonar (3).

A escolha dos três sítios de punção permitiu espaço adequado de manipulação do instrumental cirúrgico, com distância apropriada para a realização da reposição das vísceras abdominais e da síntese do diafragma. As manobras de reposicionamento das vísceras abdominais e de síntese diafragmática foram realizadas em sete dos oito cães deste grupo. Em um dos animais, fez-se necessária a conversão do procedimento toracoscópico numa toracotomia lateral, devido à impossibilidade de reposicionamento hepático para o interior da cavidade abdominal. Mesmo a ampliação da incisão do diafragma e o posicionamento do animal em "Trendelenburg" reverso mostraram-se insuficientes em razão de três lobos hepáticos que revelaram sinais de ingurgitamento e de friabilidade acentuada.

Durante as manobras de redução visceral, utilizou-se o posicionamento de "Trendelenburg" reverso em três cães. Em dois deles, a incisão de abertura diafragmática foi ampliada longitudinalmente 

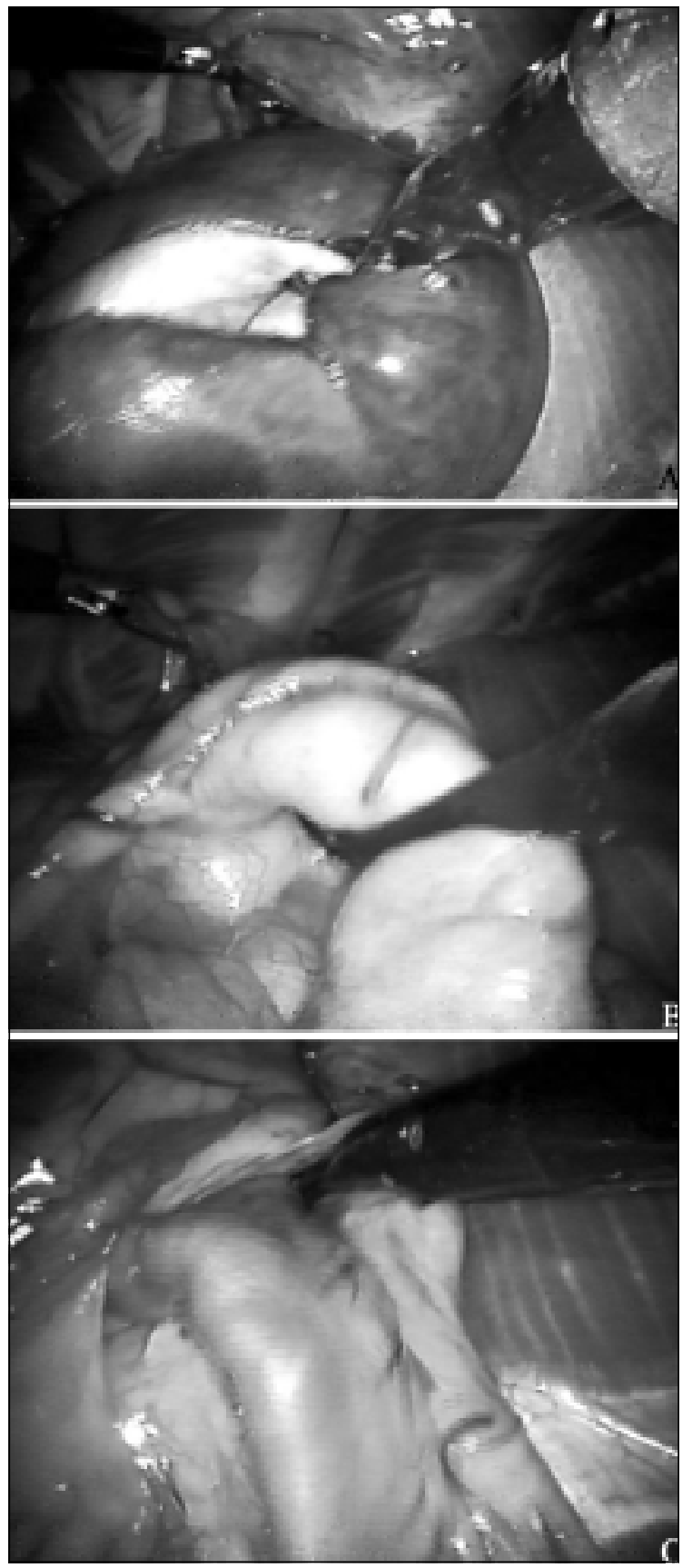

Figura 2 - Imagem toracoscópica revelando a ocorrência de hérnias diafragmáticas: com o deslocamento visceral do fígado (A), estômago (B) e intestino delgado (C).

cerca de 2 ou $3 \mathrm{~cm}$. O fígado foi o órgão que sofreu deslocamentos mais freqüientes (sete cães), variando apenas no número de lobos que ocuparam o tórax. Também quanto ao reposicionamento das vísceras deslocadas para o interior do abdome foi o fígado o órgão que apresentou maiores dificuldades.
O fio poliglactina 910, no 3-0 agulhado, previamente medido em $20 \mathrm{~cm}$, mostrou-se adequado tanto pela facilidade de manipulação no interior do tórax quanto pela resistência na realização da síntese da ruptura do diafragma. O padrão de sutura contínua simples revelou-se igualmente satisfatório, permitindo boa aposição das bordas da ferida diafragmática, além de assegurar a resistência desejada.

\section{DISCUSSÃO}

O planejamento preliminar na distribuição de equipamentos e da equipe cirúrgica, destacado por FREEMAN (1998) e BRUN (1999), mostrou-se indispensável no presente estudo, permitindo adequado espaço de trabalho com boa visibilidade do monitor e correto fluxo de pessoal no interior da sala cirúrgica. O protocolo anestésico utilizado no experimento foi considerado adequado, viabilizando a totalidade das cirurgias realizadas. $\mathrm{O}$ protocolo não incluiu a ventilação seletiva, considerada técnicapadrão para as CTVAs realizadas na cirurgia humana (JACKLITSCH et al., 1996; FAUNT et al., 1998). A opção pela ventilação pulmonar bilateral decorreu da impossibilidade de adaptar sondas desenhadas para humanos e da indisponibilidade de sondas específicas para cães no mercado - aspecto relacionado por FAUNT et al. (1998) como limitante na adoção da intubação seletiva nessa espécie.

A tentativa de desenvolver um modelo de hérnia diafragmática por meio do acesso laparoscópico, demonstrou-se efetiva, verificando-se o deslocamento de vísceras e/ou estruturas abdominais nos oito cães do experimento, sem a ocorrência de óbitos no trans e no pós-operatório. ZIEROLD et al. (2001) utilizaram o acesso toracoscópico para estabelecer uma ruptura diafragmática em suínos com o objetivo de comparar os exames de ultra-sonografia e de radiografia como métodos de diagnóstico. Os autores relacionaram dois óbitos, nos seis animais preparados experimentalmente.

Antes de promover o defeito diafragmático nos cães, optou-se por reduzir a pressão do pneumoperitônio de 11 para $5 \mathrm{mmHg}$. A manobra teve como objetivo minimizar as chances de ocorrência de um pneumotórax compressivo. Conforme as afirmações de MARCUS et al. (1996), as principais alterações do pneumotórax produzido durante uma cirurgia videoendoscópica referem-se ao sistema respiratório.

O sucesso alcançado pelos acessos videoendoscópicos, no reparo das hérnias diafragmáticas de origem traumática na medicina humana, está relacionado aos critérios de inclusão dos pacientes (LOWDERMILK \& NAUNHEIM, 2000). 
MARTINEZ et al. (2001) destacam a estabilidade hemodinâmica e a ausência de achados clínicos ou radiológicos que indiquem uma toracotomia ou celiotomia de urgência. No presente estudo, todos os cães submetidos à segunda etapa operatória apresentavam estabilidade hemodinâmica, sendo que apenas dois animais manifestaram sinais leves de dispnéia. Em ambos os casos, constatou-se o deslocamento parcial do estômago e do fígado, o que causou atelectasia de lobos pulmonares, aspecto destacado como freqüente nas hérnias diafragmáticas de cães e gatos por HAGE \& IWASAKI (2001).

No atual estudo, a utilização diagnóstica do acesso toracoscópico durante a segunda etapa do experimento permitiu a identificação da ruptura diafragmática e do deslocamento das vísceras e estruturas abdominais em todos os cães, sem diferença quanto ao lado do defeito. LODERMILK \& NAUNHEIM (2000) relacionaram um total de 15 estudos com 199 pacientes humanos submetidos à CTVA por suspeita de ruptura diafragmática, tendo o acesso alcançado índice de $98 \%$ de eficácia no diagnóstico.

A redução do conteúdo herniado em sete dos oito cães foi certamente facilitada pelo intervalo de uma semana observado entre a primeira e a segunda etapas cirúrgicas do experimento. $\mathrm{O}$ intervalo proposto limita a formação de aderências maduras com maiores níveis de tecido fibrovascular organizado, que iniciase, conforme JOHNSON (1993), a partir da segunda semana após o trauma.

\section{CONCLUSÕES}

O acesso cirúrgico videotoracoscópico é eficaz no diagnóstico de hérnias diafragmáticas em cães, porém seu êxito está condicionado à sua utilização no hemitórax correspondente à ruptura.

O acesso videotoracoscópico é viável para a realização das manobras de reposicionamento das estruturas abdominais e síntese diafragmática, em hérnias diafragmáticas com até uma semana de existência, em cães clinicamente estáveis.

\section{REFERÊNCIAS BIBLIOGRÁFICAS}

BOUDRIEAU, R.J.; MUIR, W.W. Pathophysiology of traumatic diaphragmatic hérnia in dogs. Comp Cont Educ Pract Vet, v.9, n.4, p.379-385, 1987.

BRUN, M.V. et al. Redução de hérnia diafragmática em caninos por toracoscopia. In: CONGRESSO BRASILEIRO DE CIRURGIA E ANESTESIOLOGIA VETERINÁRIA, 3., 1998, Belo Horizonte. Anais... Belo Horizonte : CBCAV, 1998. p.94.
BRUN, M.V. Ovário-histerectomia em caninos por cirurgia laparoscópica. 1999. 181f. Dissertação (Mestrado em Cirurgia Veterinária) - Curso de Pós-graduação em Cirurgia Experimental da Faculdade de Veterinária da Universidade Federal do Rio Grande do Sul.

CARRILLO, E.H. et al. Video-assisted thoracic surgery in trauma patients. Journal of the American College of Surgeons, v.184, p.316-323, 1997.

CHUNG, D.A.; RITCHIE, A.J. Videothoracoscopic drainage of mediastinal abscess: an alternative to thoracoscopy. Ann Thorac Surg, v.69, p.1573-1574, 2000.

FAUNT, K.K. et al. Evaluationof biopsy specimens obtained during thoracoscopy from lung of clinically normal dogs. Am J Vet Res, v.59, n.11, p.1499-502, 1998.

FREEMAN, L.J. Introduction. In: FREEMAN, L.J. Veterinary endosurgery. St Louis : Mosby, 1998. p.xx-xvii

GARCÍA, F. et al. Examination of the thoracic cavity and lung lobectomy by means of thoracoscopy in dogs. Can Vet J, v.39, p.285-291, 1998.

HAGE, M.C.F.N.S.; IWASAKI, M. Contribuição ao estudo radiográfico das rupturas diafragmáticas em cães e gatos. Clínica Veterinária, São Paulo, n.35, p.36-50, 2001.

JAKLITSCH, M.T. et al. Video-assited techniques in thoracic surgery. In: LOUGHLIN, K.R.; BROOKS, D.C. Principles of endosurgery. Cambridge : Blackwell Science, 1996. 255p. p.230-250.

JOHNSON, K.A. Diaphragmatic, pericardial, and hiatal hernia. In SLATTER, D. Textbook of small animal surgery. 2.ed. Philadelphia : Saunders, 1993. V.1, p.455-470.

KIRK, R.W.; BISTNER, S.I. Manual de procedimentos e tratamento de emergência em medicina veterinária. 3.ed. São Paulo : Manole, 1987. 994p.

LEVINE, S.H. Diaphragmatic hernia. Vet Clin North Amer: Small Anim Pract, Philadelphia, v.17, n.2, p.411-430, 1987.

LOWDERMILK, G.A.; NAUNHEIM, K.S. Thoracoscopic evaluation and treatment of thoracic trauma. Surg Clin North Am, Philadelphia, v.80, n.5, p.1535-1542, 2000.

MACK, M.J. et al. Present role of thoracoscopy in the diagnosis and treatment of diseases of the chest. Ann Thorac Surg, Philadelphia, v.54, p.403-409, 1992.

MARCUS, D.R. et al. Carbon dioxide pneumothorax in laparoscopic surgery. American Journal Surgery, v.171, n.5, p.464-466, 1996

MARTINEZ, M. Surg Endosc, v.15, p.28-32, 2001

MCCARTHY, T.C.; MCDERMID, S.L. Thoracoscopy. Vet Clin North Amer: Small Anim Pract, Philadelphia, v.20, n.5, p.1341-1352, 1990.

NAKAJIMA, J. et al. Thoracoscopic resection of the pulmonary aspergilloma. Chest, Chicago, v.118, n.5, p.1490-1495, 2000.

RAISER, A.G. Hérnias em cães e gatos - análise de 188 casos e proposição de técnica de herniorrafia 
para ruptura costal do diafragma. 1993. 108f. Tese (Concurso para Professor Titular) - Universidade Federal de Santa Maria.

RAISER, A.G. Herniorrafia diafragmática em cães e gatos. Relato de 22 casos e proposição de técnica para corrigir rupturas freno-costais. Braz J Vet Res Anim Sci, São Paulo, v.31, n.3/4, p.245-251, 1994

REMEDIOS, A.M.; FERGUSON, J. Minimally invasive surgery: laparoscopy and thoracoscopy in small animals. Comp Cont Educ Pract Vet, Toronto, v.18, n.11, p.11911199, 1996.

SMITH, R.S. et al. Preliminary report on videothoracoscopy in the evaluation and treatment of thoracic injury. Am J Surg, New York, v.166, p.690-695, 1993.
WALSH, P.J. et al. Thoracoscopic versus open partial pericardectomy in dogs: comparison of postoperative pain and morbidity. Vet Surg, Philadelphia, v.28, p.472-479, 1999.

WALTON, R.S. Video-assisted thoracoscopy. Vet Clin North Amer: Small Anim Pract, Philadelphia, v.31, n.4, p.729-759, 2001.

WILSON, G.P. et al. A review of 116 diaphragmatic hérnias in dogs and cats. J Amer Vet Med Assoc, v.159, n.9, p.1142$1145,1971$.

ZIEROLD, D. et al. Penetrating trauma to the diaphragm. Natural history and ultrasonographic characteristics of untreated injury in a pig model. Arch Surg, v.136, p.32-37, 2001 . 